\title{
WEAK SOLUTIONS OF THE GELLERSTEDT AND THE GELLERSTEDT-NEUMANN PROBLEMS
}

BY

\author{
A. K. AZIZ AND M. SCHNEIDER
}

ABSTRACT. We consider the question of existence of weak and semistrong solutions of the Gellerstedt problem

$$
\left.u\right|_{\Gamma_{0} \cup \Gamma_{1} \cup \Gamma_{2}}=0
$$

and the Gellerstedt-Neumann problem

$$
\left(d_{n} u=k(y) u_{x} d y-\left.u_{y} d x\right|_{\Gamma_{0}}=0,\left.\quad u\right|_{\Gamma_{1} \cup \Gamma_{2}}=0\right)
$$

for the equation of mixed type

$$
L[u] \equiv k(y) u_{x x}+u_{y y}+\lambda u=f(x, y), \quad \lambda=\text { const }<0
$$

in a region $G$ bounded by a piecewise smooth curve $\Gamma_{0}$ lying in the half-plane $y>0$ and intersecting the line $y=0$ at the points $A(-1,0)$ and $B(1,0)$. For $y<0, G$ is bounded by the characteristic curves $\gamma_{1}(x<0)$ and $\gamma_{2}(x>0)$ of (1) through the origin and the characteristics $\Gamma_{1}$ and $\Gamma_{2}$ through $A$ and $B$ which intersect $\gamma_{1}$ and $\gamma_{2}$ at the points $P$ and $Q$, respectively. Using a variation of the energy integral method, we give sufficient conditions for the existence of weak and semistrong solutions of the boundary value problems (Theorems 4.1, 4.2, 5.1).

1. Introduction. We consider the question of existence of weak solutions for the following boundary value problems of mixed type. Let

$$
L[u] \equiv k(y) u_{x x}+u_{y y}+\lambda u=f(x, y),
$$

in a bounded simply connected region $G$ in $\mathbf{R}^{2}$, where

$$
\begin{gathered}
\operatorname{sgn} k(y)=\operatorname{sgn} y, \quad k(y) \in C^{0}(\bar{G}) \cap C^{2}(\bar{G} \cap\{y<0\}), \\
\lambda=\text { constant }<0, \quad f(x, y) \in L^{2}(G) .
\end{gathered}
$$

The region $G$ is bounded by a piecewise smooth curve $\Gamma_{0}$ lying in the half-plane $y>0$ and intersecting the line $y=0$ at the points $A(-1,0)$ and $B(1,0)$. For $y<0, G$ is bounded by the characteristic curves $\gamma_{1}(x<0)$ and $\gamma_{2}(x>0)$ of $(1.1)$ through the origin and the characteristics $\Gamma_{1}$ and $\Gamma_{2}$ through $A$ and $B$ which intersect $\gamma_{1}$ and $\gamma_{2}$ at points $P$ and $Q$, respectively.

The boundary value problems treated in this paper may be stated as

$$
L(u)=f \quad \text { in } G,\left.\quad u\right|_{\Gamma_{0} \cup \Gamma_{1} \cup \Gamma_{2}}=0,
$$

$$
L(u)=f \quad \text { in } G, \quad d_{n} u=k(y) u_{x} d y-\left.u_{y} d x\right|_{\Gamma_{0}}=0,\left.\quad u\right|_{\Gamma_{1} \cup \Gamma_{2}}=0 .
$$

Received by the editors January 20, 1983 and, in revised form, August 26, 1983.

1980 Mathematics Subject Classification. Primary 35D05; Secondary 35M05. 
In the literature many authors have dealt with the question of existence and uniqueness of classical solutions for the boundary value problem (1.3) (e.g. $[\mathbf{3}, \mathbf{4}, \mathbf{5}$, 7]). In [6] sufficient conditions are given for the existence of a weak solution of (1.3) for the special case when $\lambda=0$ and the curves $\Gamma_{1}$ and $\Gamma_{2}$ are not characteristic. In the present paper, using a variation of the well-known energy integral method, we give sufficient conditions for the existence of weak solutions to boundary value problems (1.3) and (1.4). We also briefly indicate how to obtain sufficient conditions for the existence of semistrong solutions for our boundary value problems.

\section{Mathematical preliminaries. Let}

$$
W^{m, 2}(G)=\left\{u\left|u \in L^{2}(G), D^{\alpha} u \in L^{2}(G),\right| \alpha \mid \leqslant m\right\}
$$

be the Sobolev space with norm $\|\cdot\|_{m}$ and inner product $(\cdot, \cdot)_{m}$. Following [2, p. 79], we denote by $W_{(G)}^{2,2}(G, \mathrm{bd})$ the closure of the function space

$$
\tilde{W}_{(G)}^{2,2}(G, \mathrm{bd})=\left\{u\left|u \in C^{2}(\bar{G}), u\right|_{\Gamma_{0} \cup \Gamma_{1} \cup \Gamma_{2}}=0\right\}
$$

with respect to the norm $\|\cdot\|_{2}$. We associate with the formal adjoint operator

$$
L^{+} v:=k(y) v_{x x}+v_{y y}+\lambda v
$$

of $L$ the adjoint function space

$$
W_{(G)}^{2.2}\left(G, \mathrm{bd}^{+}\right)=\left\{v \mid v \in W^{2.2}(G),(l u, v)_{0}=\left(u, L^{+} v\right)_{0}, \forall u \in W_{(G)}^{2.2}(G, \mathrm{bd})\right\},
$$

or, equivalently, we may define the space $W_{(G)}^{2.2}\left(G, \mathrm{bd}^{+}\right)$as the closure of the function space

$$
\tilde{W}_{(\bar{G})}^{2,2}\left(G, \mathrm{bd}^{+}\right)=\left\{v\left|v \in C^{2}(\bar{G}), v\right|_{\Gamma_{0} \cup \gamma_{2} \cup \gamma_{2}}=0\right\},
$$

with respect to the norm $\|\cdot\|_{2}$.

Similarly, for the Gellerstedt-Neumann problem, we define the space $W_{(N)}^{2,2}(G, \mathrm{bd})$ as the closure of the function space

$$
\tilde{W}_{(N)}^{2,2}(G, \mathrm{bd})=\left\{u\left|u \in C^{2}(\bar{G}), d_{n} u\right|_{\Gamma_{0}}=0,\left.u\right|_{\Gamma_{1} \cup \Gamma_{2}}=0\right\},
$$

with respect to the norm $\|\cdot\|_{2}$. Analogously, the adjoint space $W_{(N)}^{2.2}\left(G, \mathrm{bd}^{+}\right)$is defined as the closure of the function space

$$
\tilde{W}_{(N)}^{2,2}\left(G, \mathrm{bd}^{+}\right)=\left\{v\left|v \in C^{2}(\bar{G}), d_{n} v\right|_{\Gamma_{0}}=0,\left.v\right|_{\gamma_{1} \cup \gamma_{2}}=0\right\},
$$

with respect to the norm $\|\cdot\|_{2}$.

A function $u \in L^{2}(G)$ is said to be a weak solution of (1.3) if

$$
\left(u, L^{+} v\right)_{0}=(f, v)_{0}, \quad \forall v \in w_{(G)}^{2,2}\left(G, \mathrm{bd}^{+}\right)
$$

Similarly, $u \in L^{2}(G)$ is a weak solution of (1.4) if

$$
\left(u, L^{+} v\right)_{0}=(f, v)_{0}, \quad \forall v \in w_{(N)}^{2,2}\left(G, \mathrm{bd}^{+}\right) .
$$

It is known $[2,8]$ that a necessary and sufficient condition for the existence of a weak solution of (1.3) and (1.4) is that the following inequalities hold.

$$
\begin{aligned}
& \left\|L^{+} v\right\|_{0} \geqslant C_{1}\|v\|_{0}, \quad \forall v \in w_{(G)}^{2,2}\left(G, \mathrm{bd}^{+}\right) . \\
& \left\|L^{+} v\right\|_{0} \geqslant c_{2}\|v\|_{0}, \quad \forall v \in w_{(N)}^{2,2}\left(G, \mathrm{bd}^{+}\right),
\end{aligned}
$$


where $c_{i}, i=1,2$, in the above inequalities denote positive constants. A similar set of inequalities provide necessary and sufficient conditions for the existence of semistrong solutions [2, p. 80] of (1.3) and (1.4). Namely, if in addition to inequalities (2.1) and (2.2), the inequalities

$$
\begin{array}{ll}
\|L u\|_{0} \geqslant c_{3}\|u\|_{0}, \quad \forall u \in w_{(G)}^{2.2}(G, \mathrm{bd}), & c_{3}>0, \\
\|L u\|_{0} \geqslant c_{4}\|u\|_{0}, \quad \forall u \in w_{(N)}^{2,2}(G, \mathrm{bd}), & c_{4}>0,
\end{array}
$$

are satisfied.

In the following sections we show that, under suitable hypotheses, (2.1)-(2.4) hold for (1.3) and (1.4).

3. A priori estimates. For convenience we write the differential operator (1.1) in the form

$$
L(u) \equiv\left(A^{i k} u_{x i}\right)_{x k}+\lambda u, \quad i=1,2,
$$

where

$$
A^{\pi}=k(y), \quad A^{12}=A^{21}=0, \quad A^{22}=1 .
$$

Let

$$
\begin{gathered}
\tau=\left(x_{1}, x_{2}\right)=(x, y), \quad G^{+}=G \cap\{y>0\}, \quad G^{-}=G \cap\{y<0\}, \\
G_{1}^{-}=\bar{G} \cap\{x<0\}, \quad G_{2}^{-}=\bar{G} \cap\{x>0\} .
\end{gathered}
$$

The repeated indices as a subscript or superscript denote a summation over $i, k=1,2$. Let $\alpha^{0}(\tau), \alpha^{i}(\tau)$ be real-valued functions (to be determined) such that

$$
\alpha^{0} \in C^{2}\left(\bar{G}^{+}\right) \cap C^{2}\left(\bar{G}_{1}^{-}\right) \cap C^{2}\left(\bar{G}_{2}^{-}\right), \quad \alpha^{i}(\tau) \in C^{1}\left(\bar{G}^{+}\right) \cap C^{1}\left(\bar{G}_{1}^{-}\right) \cap C^{1}\left(\bar{G}_{2}^{-}\right) .
$$

We denote

$$
\lim _{y \rightarrow 0_{+}} \alpha^{i}=\alpha_{+}^{i}, \quad \lim _{y \rightarrow 0_{-}} \alpha^{i}=\alpha_{-}^{i}, \quad l u=\alpha^{0} u+\alpha^{1} u_{x}+\alpha^{2} u_{y} .
$$

Using the identity

$$
u_{x_{k}} u_{x_{i} x_{j}}=\left(u_{x_{k}} u_{x_{i}}\right)_{x_{j}}-u_{x_{i}} u_{x_{k} x_{j}},
$$

by a simple calculation we deduce the identity

$$
2(l u)(L(u))=P_{x}^{1}+P_{x}^{2}-\left(a_{00}\right) u^{2}-\left(a_{11} u_{x}^{2}+2 a_{12} u_{x} u_{y}+a_{22} u_{y}^{2}\right),
$$

where

$$
\begin{aligned}
& P^{1}=2 k(y) u_{x}(l u)+\alpha^{1}\left(\lambda u^{2}-k(y) u_{x}^{2}-u_{y}^{2}\right)-u^{2} k(y) \alpha_{x}^{0}, \\
& P^{2}=2 u_{y}(l u)+\alpha^{2}\left(\lambda u^{2}-k(y) u_{x}^{2}-u_{y}^{2}\right)-u^{2} \alpha_{y}^{0}, \\
& a_{00}=-2 \alpha^{0} \lambda+\lambda \alpha_{x}^{1}+\lambda \alpha_{y}^{2}=k(y) \alpha_{x x}^{0}-\alpha_{y y}^{0}, \\
& a_{11}=k(y)\left(\alpha_{x}^{1}-\alpha_{y}^{2}\right)-\alpha^{2} k^{\prime}(y)+2 \alpha^{0} k(y), \\
& a_{12}=a_{21}=\alpha_{y}^{1}+k(y) \alpha_{x}^{2}, \\
& a_{22}=-\left(\alpha_{x}^{1}-\alpha_{y}^{2}\right)+2 \alpha^{0} .
\end{aligned}
$$


By the application of Green's theorem, from (3.4) we obtain

$$
\begin{aligned}
& \iint_{G^{+} \cup \bar{G}} 2(l u) L(u) d x d y+\iint_{G^{+} \cup \bar{G}}\left(a_{00} u^{2}+a_{11} u_{x}^{2}+2 a_{12} u_{x} u_{y}+a_{22} u_{y}^{2}\right) d x d y \\
& =\int_{\partial G^{+} \cup \partial \bar{G}} P^{i} \mathbf{n}_{i} d s \\
& =\int_{\partial G} P^{1} d y-P^{2} d x \\
& \quad+\int_{-1}^{1}\left\{-\left(\alpha_{+}^{2}-\alpha_{-}^{2}\right) u_{y}^{2}-2\left(\alpha_{+}^{1}-\alpha_{-}^{1}\right) u_{x} u_{y}\right. \\
& \left.\quad-2\left(\alpha_{+}^{0}-\alpha_{-}^{0}\right) u u y+u^{2}\left[-\left(\alpha_{+}^{2}-\alpha_{-1}^{2}\right) \lambda+\left(\alpha_{y+}^{0}-\alpha_{y-}^{0}\right)\right]\right\} d x
\end{aligned}
$$

where $\mathbf{n}=\left(n_{1}, n_{2}\right)$ denotes the outer normal to $\partial G$. From (3.6) with appropriate choices of the functions $\alpha^{0}, \alpha^{i}, i=1,2$, we obtain

THEOREM 3.1. If $\rho_{i}, i=1,2$, are arbitrary postive real numbers and

$$
\begin{gathered}
\alpha_{+}^{2}-\alpha_{-}^{2} \leqslant 0, \quad \alpha_{+}^{1}-\alpha_{-}^{1}=0, \quad \alpha_{+}^{0}-\alpha_{-}^{0}=0, \\
-\left(\alpha_{+}^{2}-\alpha_{-}^{2}\right) \lambda+\left(\alpha_{y+}^{0}-\alpha_{y-}^{0}\right) \geqslant 0, \\
\int_{\partial G} P^{1} d y-P^{2} d x \geqslant 0, \\
A_{11}=-a_{11}-\rho_{1}\left(\alpha^{1}\right)^{2} \geqslant 0, \quad A_{12}=-a_{12}-\rho_{1} \alpha^{1} \alpha^{2}, \\
A_{22}=-a_{22}-\rho_{1}\left(\alpha^{2}\right)^{2} \geqslant 0, \quad A_{11} A_{22}-A_{12}^{2} \geqslant 0, \\
A_{00}=-a_{00}-\rho_{2}\left(\alpha^{0}\right)^{2} \geqslant d_{0}>0
\end{gathered}
$$

in $\bar{G}$, then we have

$$
\|u\|_{0} \leqslant c\|L u\|_{0}, \quad c>0, \quad \forall u \in c^{2}(\bar{G}) .
$$

If , in addition, $A_{11} \cdot A_{22}-A_{12}^{2} \geqslant d_{1}>0$ in $\bar{G}$, we have

$$
\|u\|_{1} \leqslant c\|L u\|_{0}, \quad \forall u \in c^{2}(\bar{G}) \text {. }
$$

Proof. With hypotheses (3.7) and (3.8), from (3.6) we have

$$
-\iint_{G^{+} \cup G^{-}}\left\{a_{00} u^{2}+a_{11} u_{x}^{2}+2 a_{12} u_{x} u_{y}+a_{22} u_{y}^{2}\right\} d x d y \leqslant \int_{G^{+} \cup G^{-}} 2(l u) f d x d y .
$$

Using the inequality

$$
2|a b| \leqslant \rho a^{2}+b^{2} / \rho, \quad \rho>0,
$$


we obtain for the integral on the right side of (3.10) the estimate

$$
\begin{array}{r}
\left|\iint_{G} 2 f(l u) d x d y\right| \\
\leqslant \iint_{G}\left\{\frac{1}{\rho_{1}} f^{2}+\rho_{1}\left[\left(\alpha^{1}\right)^{2} u_{x}^{2}+2 \alpha^{1} \alpha^{2} u_{x} u_{y}+\left(\alpha^{2}\right)^{2} u_{y}^{2}\right]\right. \\
\left.+\frac{1}{\rho_{2}} f^{2}+\rho_{2}\left(\alpha^{0}\right)^{2} u^{2}\right\} d x d y .
\end{array}
$$

Thus it follows that

(3.11) $\iint_{G}\left\{A_{11} u_{x}^{2}+2 A_{12} u_{x} u_{y}+A_{22} u_{y}^{2}+A_{00} u^{2}\right\} d x d y \leqslant\left(\frac{1}{\rho_{2}}+\frac{1}{\rho_{2}}\right)\|L u\|_{0}^{2}$.

By hypothesis (3.9) we conclude that

$$
\|u\|_{0} \leqslant c\|L u\|_{0} \text {. }
$$

If, in addition, we have $A_{11} A_{22}-A_{12}^{2} \geqslant d_{1}>0$ in $\bar{G}$, it follows that

$$
\begin{aligned}
& A_{11} u_{x}^{2}+2 A_{12} u_{x} u_{y}+A_{22} u_{y}^{2} \\
& \quad \geqslant \frac{A_{11}+A_{22}}{2}\left[1-\sqrt{1-\frac{4\left(A_{11} A_{22}-A_{12}^{2}\right)}{\left(A_{11}+A_{22}\right)^{2}}}\right]\left(u_{x}^{2}+u_{y}^{2}\right) . \\
& \geqslant \mu_{0}\left(u_{x}^{2}+u_{y}^{2}\right), \quad \mu_{0}>0 .
\end{aligned}
$$

Thus

$$
\|u\|_{1} \leqslant c\|L u\|_{0}
$$

In order to make use of these estimates in dealing with our boundary value problems, we need to choose $\alpha^{0}, \alpha^{i}, i=1,2$, so that estimates (3.7) and (3.9) hold, and the boundary integral $\int_{\partial G} P^{1} d y-P^{2} d x$ is nonnegative. We first consider this integral on $\Gamma_{0}$, where $\mu=0$ is equivalently $u_{x} d x+u_{y} d y=0$ on smooth pieces of $\Gamma_{0}$. Thus we have

(3.13) $2 k(y) \alpha^{2} u_{x} u_{y} d y=-2 k(y) \alpha^{2} u_{x}^{2} d x, \quad 2 \alpha^{1} u_{x} u_{y} d x=-2 \alpha^{1} u_{y}^{2} d y$.

From the definition of $P^{1}$ and $P^{2}$ (see (3.5)) and (3.13) it follows that

$$
\int_{\Gamma_{0}} P^{1} d y-P^{2} d x=\int_{\Gamma_{0}}\left[k(y) u_{x}^{2}+u_{y}^{2}\right]\left[\alpha^{1} d y+\alpha^{2} d x\right]
$$

If on $\Gamma_{0}$ we have the boundary condition $d_{n} u=k(y) u_{x} d y-u_{y} d x=0$, then

$$
\begin{aligned}
\int_{\Gamma_{0}} P^{1} d y-P^{2} d x= & \int_{\Gamma_{0}} u^{2}\left\{\lambda\left(\alpha^{1} d y-\alpha^{2} d x\right)-\left(k(y) \alpha_{x}^{0} d y-\alpha_{y}^{0} d x\right)\right\} \\
& -\int_{\Gamma_{0}}\left(k(y) u_{x}^{2}+u_{y}^{2}\right)\left(\alpha^{1} d y-\alpha^{2} d x\right)
\end{aligned}
$$


Since $\Gamma_{1}$ and $\gamma_{2}$ are characteristics of $(1.1)$, we have the relation $(-k(y))^{1 / 2} d y+d x$ $=0$, which implies

$$
\begin{aligned}
2 \alpha^{0} u\left(k(y) u_{x} d y-u_{y} d x\right) & =2 \alpha^{0} u\left(-(-k)^{1 / 2}(-k)^{1 / 2} u_{x} d y-u_{y} d x\right) \\
& =2 \alpha^{0} u(-k)^{1 / 2} d u
\end{aligned}
$$

Integrating both sides of (3.16), we have

$$
\int_{\Gamma_{1}, \gamma_{2}} 2 \alpha^{0}\left(k(y) u_{x} d y-u_{y} d x\right)=\left.\alpha^{0}(-k)^{1 / 2} u^{2}\right|_{A, 0} ^{P, Q} \int_{\Gamma_{1}, \gamma_{2}} u^{2} d\left[\alpha^{0}(-k)^{1 / 2}\right]
$$

Now using the fact that

$$
\begin{gathered}
\left(\alpha^{1} k(y) u_{x}^{2}-\alpha^{1} u_{y}^{2}+2 \alpha^{2} k(y) u_{x} u_{y}\right) d y+\left(\alpha^{2} k(y) u_{x}^{2}-\alpha^{2} u_{y}^{2}-2 \alpha^{1} u_{x} u_{y}\right) d x \\
=-\left[(-k)^{1 / 2} u_{x}-u_{y}\right]^{2}\left(\alpha^{1} d y+\alpha^{2} d x\right),
\end{gathered}
$$

we obtain

$$
\begin{aligned}
\int_{\Gamma_{1}, \gamma_{2}} P^{1} d y & -P^{2} d x=\left.\alpha^{0}(-k)^{1 / 2} u^{2}\right|_{A, 0} ^{P, Q} \int_{\Gamma_{1}, \gamma_{2}}\left[(-k)^{1 / 2} u_{x}-u_{y}\right]^{2}\left(\alpha^{1} d y+\alpha^{2} d x\right) \\
& +\int_{\Gamma_{1}, \gamma_{2}} u^{2}\left\{-d\left[\alpha^{0}(-k)^{1 / 2}\right]+\left(\alpha^{1} \lambda-k(y) \alpha_{x}^{0}\right) d y+\left(-\alpha^{2} \lambda+\alpha_{y}^{0}\right) d x\right\} .
\end{aligned}
$$

In a similar way, noting that the characteristics $\Gamma_{2}, \gamma_{1}$ satisfy the relation $(-k)^{1 / 2} d y$ $-d x=0$, we obtain

$$
\begin{aligned}
\int_{\Gamma_{2}, \gamma_{1}} P^{1} d y & -P^{2} d x=-\left.\alpha^{0}(-k)^{1 / 2} u^{2}\right|_{P, Q} ^{0, B} \int_{\Gamma_{2}, \gamma_{1}}\left[(-k)^{1 / 2} u_{x}+u_{y}\right]^{2}\left(\alpha^{1} d y+\alpha^{2} d x\right) \\
& +\int_{\Gamma_{2}, \gamma_{1}} u^{2}\left\{d\left[\alpha^{0}(-k)^{1 / 2}\right]+\left(\alpha^{1} \lambda-k(y) \alpha_{x}^{0}\right) d y+\left(-\alpha^{2} \lambda+\alpha_{y}^{0}\right) d x\right\} .
\end{aligned}
$$

4. Existence of solutions for the Gellerstedt problem. We recall that in order to prove the existence of a weak solution for problem (1.3) it is sufficient to show that

$$
\left\|L^{+} v\right\|_{0} \geqslant c\|v\|_{0}, \quad \forall v \in w_{G}^{2,2}\left(G, \mathrm{bd}^{+}\right) .
$$

We observe that the characteristics for $L^{+}$satisfy the equations

$$
\sqrt{-k} d y-\left.d x\right|_{\gamma_{1}, \Gamma_{2}}=0, \quad \sqrt{-k} d y+\left.d x\right|_{\Gamma_{1}, \gamma_{2}}=0 .
$$

Using the above equations and recalling that the functions in $w_{G}^{2.2}\left(G, \mathrm{bd}^{+}\right)$satisfy the boundary condition $\left.v\right|_{\gamma_{1} \cup \gamma_{2}}=0$, we have $\int_{\gamma_{1}, \gamma_{2}} P^{1} d x-P^{2} d y=0$. Now from Theorem 3.1 and (3.17), (3.18) it follows that

$$
\left\|L^{+} v\right\|_{0} \geqslant c\|v\|_{0}, \quad \forall v \in w_{G}^{2,2}\left(G, \mathrm{bd}^{+}\right),
$$

provided the functions $\alpha^{0}(\tau), \alpha^{i}(\tau), i=1,2$, satisfy the following conditions in $G$. 
$\mathrm{C}_{1}: \operatorname{In} G^{+} \equiv G \cap\{y>0\}$ we must have

$$
\begin{aligned}
& \alpha^{1} d y+\left.\alpha^{2} d x\right|_{\Gamma_{0}} \geqslant 0, \\
& A_{11}=-k(y)\left(\alpha_{x}^{1}-\alpha_{y}^{2}\right)+\alpha^{2} k^{\prime}(y)-2 \alpha^{0} k(y)-\rho_{1}\left(\alpha^{1}\right)^{2} \geqslant 0, \\
& A_{12}=-k(y) \alpha_{x}^{2}-\alpha_{y}^{1}-\rho_{1} \alpha^{1} \alpha^{2}, \\
& A_{22}=\left(\alpha_{x}^{1}-\alpha_{y}^{2}\right)-2 \alpha^{0}-\rho_{1}\left(\alpha^{2}\right)^{2} \geqslant 0, \\
& A_{00}=k(y) \alpha_{x x}^{0}+\alpha_{y y}^{0}+2 \lambda \alpha^{0}-\lambda \alpha_{x}^{1}-\lambda \alpha_{y}^{2}-\rho_{2}\left(\alpha^{0}\right)^{2} \geqslant d_{0}>0, \\
& A_{11} A_{22}-A_{12}^{2} \geqslant 0 .
\end{aligned}
$$

$\mathrm{C}_{2}:$ On $G \cap\{y=0\}$,

$$
\begin{gathered}
\alpha_{+}^{2}-\alpha_{-}^{2} \leqslant 0, \quad \alpha_{+}^{1}-\alpha_{-}^{1}=0, \quad \alpha_{+}^{0}-\alpha_{-}^{0}=0, \\
-\left(\alpha_{+}^{2}-\alpha_{-}^{2}\right) \lambda+\left(\alpha_{y+}^{0}-\alpha_{y-}^{0}\right) \geqslant 0 .
\end{gathered}
$$

$\mathrm{C}_{3}$ : In $G^{-} \equiv G \cap\{y<0\}$ we need the inequalities:

$$
\begin{aligned}
& \text { on } \Gamma_{1}:\left(-\alpha^{1} d y+\alpha^{2} d x\right) \geqslant 0, \\
& 2 \sqrt{-k}\left[-\sqrt{-k} \alpha_{x}^{0}+\alpha_{y}^{0}\right]-\alpha^{0} \frac{k^{\prime}}{2 \sqrt{-k}}-\alpha^{1} \lambda-\alpha^{2} \sqrt{-k} \lambda \geqslant 0 ; \\
& \text { on } \Gamma_{2}:\left(-\alpha^{1} d y+\alpha^{2} d x\right) \geqslant 0, \\
& 2 \sqrt{-k}\left[+\sqrt{-k} \alpha_{x}^{0}+\alpha_{y}^{0}\right]-\alpha^{0} \frac{k^{\prime}}{2 \sqrt{-k}}+\alpha^{1} \lambda-\alpha^{2} \sqrt{-k} \lambda \geqslant 0 ; \\
& A_{11} \geqslant 0, \quad A_{22} \geqslant 0, \quad A_{00} \geqslant d_{1}>0, \quad A_{11} A_{22}-A_{12}^{2} \geqslant 0,
\end{aligned}
$$

where $A_{11}, A_{12}$, and $A_{00}$ are defined as in (4.1).

We write $G^{-}=G_{1}^{-} \cup G_{2}^{-}$, with $G_{1}^{-}=G^{-} \cap\{x<0\}, G_{2}^{-}=G^{-} \cap\{x>0\}$ as in $\S 3$. Now we choose the functions $\alpha^{0}(\tau), \alpha^{i}(\tau), i=1,2$, separately in $G_{1}^{-}$and $G_{2}^{-}$so (4.3) is satisfied. In $G_{1}^{-}$we impose on $\alpha^{1}$ and $\alpha^{2}$ the conditions

$$
\alpha^{1}-\sqrt{-k} \alpha^{2}=0, \quad \alpha^{2} k^{\prime}(y)-4 k(y) \alpha^{0}=0 .
$$

From (4.4) it follows that

$$
\sqrt{-k}\left(\alpha^{1}-\sqrt{-k} \alpha^{2}\right)_{x}+\left(\alpha^{1}-\sqrt{-k} \alpha^{2}\right)_{y} \equiv 0 .
$$

\section{Letting}

$$
E=\left(\alpha_{x}^{1}-\alpha_{y}^{2}-2 \alpha^{0}-\rho_{1}\left(\alpha^{2}\right)^{2}\right),
$$

$A_{11}, A_{12}$ and $A_{22}$ may be written

$$
A_{11}=-k(y) E, \quad A_{12}=\sqrt{-k} E, \quad A_{22}=E .
$$

Hence

$$
A_{11} A_{22}-A_{12}^{2} \equiv 0 \text {. }
$$

From (4.3) we must have $A_{11} \geqslant 0$ and $A_{22} \geqslant 0$; therefore we require that $E \geqslant 0$ in $G_{1}^{-}$. Using (4.4), this condition may be written as a condition on $\alpha^{0}$, namely

$$
4 \frac{\sqrt{-k}}{k}\left[-\sqrt{-k} \alpha_{x}^{0}+\alpha_{y}^{0}\right]-2 \alpha^{0}\left(1+2\left(\frac{k}{k^{\prime}}\right)^{\prime}\right)-16 \rho_{1}\left(\frac{k}{k^{\prime}} \alpha^{0}\right)^{2} \geqslant 0 .
$$


Similarly if we write the conditions (4.3) on $\Gamma_{1}$ and the condition $A_{00} \geqslant d_{0}>0$ in terms of $\boldsymbol{\alpha}^{0}$, we obtain

$$
\begin{gathered}
\sqrt{-k}\left[-\sqrt{-k} \alpha_{x}^{0}+\alpha_{y}^{0}\right]+4 \lambda \alpha{\frac{O(-k)^{3 / 2}}{k^{\prime}}}^{3}\left(1-\frac{1}{16 \lambda}\left(\frac{k^{\prime}}{k}\right)^{2}\right) \geqslant 0 \quad \text { on } \Gamma_{1}, \\
A_{00}=k(y) \alpha_{x x}^{0}+\alpha_{y y}^{0}+4 \lambda \frac{(-k)}{k^{\prime}}\left[\sqrt{-k} \alpha_{x}^{0}+\alpha_{y}^{0}\right]+2 \lambda \alpha^{0}\left(1-2\left(\frac{k}{k^{\prime}}\right)^{\prime}\right) \\
-\rho_{2}\left(\alpha^{0}\right)^{2} \geqslant d_{0}>0 .
\end{gathered}
$$

Now we choose in $G_{1}^{-}$,

$$
\alpha^{0}=\alpha^{0}(y) \text { and } \alpha^{0}(y)<0 .
$$

Since $\lambda<0$ in $\bar{G}$, with this choice of $\alpha^{0}$, from (4.8) we have

$$
\alpha_{y y}^{0}+4 \lambda\left(\frac{-k}{k^{\prime}}\right) \alpha_{y}^{0}-4 \lambda \alpha^{0}\left(\frac{k}{k^{\prime}}\right)^{\prime}=\frac{d}{d y}\left(\alpha_{y}^{0}-\frac{4 k}{k^{\prime}} \alpha^{0}\right)=d_{0}>0 .
$$

By integration (for $y_{p} \leqslant y<0\left(P=\left(-\frac{1}{2}, y_{p}\right)\right)$, we get

$$
\begin{aligned}
& \alpha^{0}(y)=\exp \left(-\int_{t=y}^{0} 4 \lambda \frac{k}{k^{\prime}} d t\right)\left\{\alpha_{00}^{0}-\int_{t=y}^{0} d_{0}(t-y)_{p} \exp \left(\int_{s=t}^{0} 4 \lambda \frac{k}{k^{\prime}} d s\right) d t\right\}<0, \\
& \alpha_{y}^{0}(y)=4 \frac{k}{k^{\prime}} \alpha^{0}+d_{0}\left(y-y_{p}\right),
\end{aligned}
$$

where $\alpha_{00}^{0}<0$ is an arbitrary constant.

A simple computation shows that with (4.11) condition (4.7) is satisfied. From (4.6) it follows that

(4.12) $-2 \alpha^{0}\left[8 \lambda\left(\frac{k}{k^{\prime}}\right)^{2}+1+2\left(\frac{k}{k^{\prime}}\right)\right]+4 \frac{(-k)}{k^{\prime}} d_{0}\left(y-y_{p}\right)-16 \rho_{1}\left(\frac{k}{k^{\prime}} \alpha^{0}\right)^{2} \geqslant 0$.

Hence if

$$
\begin{aligned}
& k^{\prime}(y)>0 \text { in } \overline{G_{1}^{-}} \cap\{y<0\}, \\
& 8 \lambda\left(\frac{k}{k^{\prime}}\right)^{2}+1+2\left(\frac{k}{k^{\prime}}\right)^{\prime}>0 \quad \text { in } G_{1}^{-}, \\
& \lim _{y \rightarrow 0-}\left(1+2\left(\frac{k}{k^{\prime}}\right)^{\prime}\right)>0, \quad \lim _{y \rightarrow 0-} \frac{k(y)}{k^{\prime}(y)}=0,
\end{aligned}
$$

then (4.12) holds and we have

$$
\alpha_{-}^{0}=\alpha_{00}^{0}(<0), \quad \alpha_{y-}^{0}=\alpha_{0} y_{p}(>0) .
$$

Now consider the choice of $\alpha^{0}, \alpha^{1}$ and $\alpha^{2}$ in $G_{2}^{-}=G^{-} \cap\{x>0\}$. In $G_{2}^{-}$we impose on $\alpha^{1}$ and $\alpha^{2}$ the conditions

$$
\alpha^{1}+\sqrt{-k} \alpha^{2}=0, \quad \alpha^{2} k^{\prime}-4 k \alpha^{0}=0
$$

which lead to

$$
\sqrt{-k} \cdot\left(\alpha^{1}+\sqrt{-k} \alpha^{2}\right)_{x}-\left(\alpha^{1}+(\sqrt{-k}) \alpha^{2}\right)_{y} \equiv 0
$$


Using (4.5) we have, as before,

$$
A_{11}=-k(y) E, \quad A_{12}=\sqrt{-k} E, \quad A_{22}=E .
$$

Hence, once again, $A_{11} A_{22}-A_{12}^{2} \equiv 0$. From $A_{11} \geqslant 0, A_{22} \geqslant 0$, we must have $E \geqslant 0$. With (4.15) this condition, conditions (4.3) on $\Gamma_{2}$ and $A_{00} \geqslant d_{0}>0$, can be written in terms of $\alpha^{0}$ as

$$
\begin{aligned}
& 4 \frac{(-k)}{k^{\prime}}\left[\sqrt{-k} \alpha_{x}^{0}+\alpha_{y}^{0}\right]-2 \alpha_{0}\left(1+2+\left(\frac{k}{k^{\prime}}\right)^{\prime}\right)-16 \rho_{1}\left(\frac{k}{k^{\prime}} \alpha^{0}\right)^{2} \geqslant 0, \\
& \sqrt{-k}\left[\sqrt{-k} \alpha_{x}^{0}+\alpha_{y}^{0}\right]+4 \lambda \alpha^{0}{\frac{(-k)}{k^{\prime}}}^{3 / 2}\left(1-\frac{1}{16 \lambda}\left(\frac{k^{\prime}}{k}\right)^{2}\right) \geqslant 0 \quad \text { on } \Gamma_{2}, \\
& A_{00}=k(y) \alpha_{x x}^{0}+\alpha_{y y}^{0}+4 \lambda \frac{(-k)}{k^{\prime}}\left[-\sqrt{-k} \alpha_{x}^{0}+\alpha_{y}^{0}\right] \\
& +2 \lambda \alpha^{0}\left(1-2\left(k / k^{\prime}\right)^{\prime}-\rho_{2}\left(\alpha^{0}\right)^{2} \geqslant d_{0}>0 .\right.
\end{aligned}
$$

Now if we choose $\alpha^{0}$ as given by (4.11) and assume (4.13) holds in $G_{2}^{-}$, conditions (4.16)-(4.18) are satisfied and (4.14) follows as before.

With $\alpha^{0}$ given by (4.11) conditions (4.4) and (4.15), with $\lim _{y \rightarrow 0}\left(k / k^{\prime}\right)=0$, imply that

$$
\alpha_{-}^{0}=\alpha_{00}^{0}(<0), \quad \alpha_{y-}^{0}=-d y_{p}>0, \quad \alpha_{-}^{1}=\alpha_{-}^{2}=0 .
$$

Thus we may choose in $G^{+}$,

$$
\alpha^{1}=0, \quad \alpha^{2}=0, \quad \alpha^{0}(y)=\left(-d_{0} y_{p}\right) y+\alpha_{00}^{0},
$$

and since $G$ is bounded, $\alpha_{00}^{0}$ can be determined so that $\alpha^{0}(y)<0$ in $G^{+}$.

For $\alpha^{0}, \alpha^{1}, \alpha^{2}$ as given by (4.19), conditions (4.1) are satisfied, i.e.,

$$
\begin{gathered}
A_{11}=-2 k(y) \alpha^{0} \geqslant 0, \quad A_{12}=0, \quad A_{22}=-2 \alpha^{0} \geqslant 0, \\
A_{00}=2 \lambda \alpha^{0}-\rho_{2}\left(\alpha^{0}\right)^{2} \geqslant d_{0}^{*}>0
\end{gathered}
$$

(with arbitrary positive constant $\rho_{2}$ chosen sufficiently small).

Now we may state our results as

THEOREM 4.1. If

(1) $\operatorname{sgn} k(y)=\operatorname{sgn} y, \quad k(y) \in C^{0}(\bar{G}) \cap C^{2}(\bar{G} \cap\{y<0\}), \quad k^{\prime}(y)>0$ in $\bar{G} \cap$ $\{y<0\}$,

$$
\lim _{y \rightarrow 0} \frac{k(y)}{k^{\prime}(y)}=0, \quad \lim _{y \rightarrow 0-}\left(1+2\left(\frac{k}{k^{\prime}}\right)^{\prime}\right)>0, \quad 8 \lambda\left(\frac{k}{k^{\prime}}\right)^{2}+1+2\left(\frac{k}{k^{\prime}}\right)^{\prime}>0
$$

in $\bar{G}=G \cap\{y<0\}$.

(2) $\lambda<0, f(x, y) \in L^{2}(G)$,

then there exists a weak solution $u \in L^{2}(G)$ of the Gellerstedt boundary value problem

$$
L(u)=k(y) u_{x}+u_{y y}+\lambda u=f(x, y) \text { in } G,\left.\quad u\right|_{\Gamma_{0} \cup \Gamma_{1} \cup \Gamma_{2}}=0 .
$$

Remark. Our a priori estimates imply the uniqueness of a strong solution (if it exists) of the boundary value problem $L^{+}(v)=f,\left.v\right|_{\Gamma_{0} \cup \gamma_{1} \cup \gamma_{2}}=0$. The essential 
restrictive assumption in Theorem 4.1 is the condition

$$
8 \lambda\left(k / k^{\prime}\right)^{2}+1+2\left(k / k^{\prime}\right)>0 \text { in } G^{-} .
$$

Agmon, Nirenberg and Protter [1] proved the uniqueness of a strong solution for the Tricomi problem by the use of a maximum principle. This proof applies equally well to the Gellerstedt problem. On p. 64 of [1] the "conditions A" are essential. If we calculate for (1.1) the condition A5 of [1], we obtain

$$
8 \lambda\left(k / k^{\prime}\right)^{2}+1 / 2+2\left(k / k^{\prime}\right) \geqslant 0 \text { in } G \cap\{y<0\} .
$$

Thus our condition is an improvement for the uniqueness of a strong solution of the problem $L^{+}(V)=f,\left.v\right|_{\Gamma_{0} \cup \gamma_{1} \cup \gamma_{2}}=0$. To establish the existence of a semistrong solution of (1.3) we need, in addition, to show that the a priori estimate

$$
\|L(u)\|_{0} \geqslant c\|u\|_{0}, \quad c>0, \quad \forall u \in w_{(G)}^{2,2}(G, \mathrm{bd})
$$

holds.

From the proof of Theorem 4.1, we see that we need only interchange $\alpha^{1}$ and $\alpha^{2}$ (in (4.4) and (4.15)) in $G_{1}^{-}$and $G_{2}^{-}$. Thus we have

THEOREM 4.2. Under the hypotheses of Theorem 4.1, the boundary value problem (1.3) has a semistrong solution. If a strong solution exists, then this solution is unique.

For the special case when $k(y)=\operatorname{sgn} y|y|^{m}, m>0$, in (1.3), from Theorems 4.1 and 4.2 we obtain

COROLlaRy 4.1 The Gellerstedt boundary value problem (1.3) with $k(y)=$ $\operatorname{sgn} y|y|^{m}, m>0, f \in L^{2}(G)$ has a semistrong solution if

$$
(-\lambda)<\frac{m+2}{8}\left[\frac{4}{m+2}\right]^{4 \wedge m+2)} m, \quad \lambda<0 .
$$

This result can be improved in case $n>2$. In this case in $G^{-}=G \cap\{y<0\}$

$$
\begin{aligned}
& \alpha^{1}=-(4 / m)(-y)^{m / 2+1}, \quad \alpha^{2}=(4 / m) \alpha^{0}, \\
& \alpha^{0}=\alpha_{00}=\text { constant }<0,
\end{aligned}
$$

and in $G^{+}$,

$$
\alpha^{1}=\alpha^{2}=0, \quad \alpha^{0}=\alpha_{00}^{0}=\text { constant }<0 .
$$

We get

COROLlary 4.2 The Gellerstedt boundary value problem (1.3) with $k(y)=$ $\operatorname{sgn} y|y|^{m}, m>2, \lambda<0, f \in L^{2}(G)$ has a semistrong solution.

5. Existence of solutions for the Gellerstedt-Neumann problem. As it is readily seen from part 3, to prove the required estimates for the Gellerstedt-Neumann boundary value problem (4.1) we need only replace (3.14) by (3.15) in $\S 4$. Using (4.4) and (4.15), however, it turns out that it is not useful to determine $\alpha^{0}(y)$ by (4.11) in $G^{-}$, because in this case we cannot continue $\alpha^{0}$ into $G^{+}$, so that on $G \cap\{y=0\}$ 
condition (4.2) is satisfied. Instead we determine $\alpha_{0}$ in $G^{-}$to be

$$
\alpha^{0}(y)=\alpha_{00}^{0} c^{-\int_{y}^{t} 4 \lambda k / k^{\prime} d t}, \quad \alpha_{00}^{0}=\text { constant }<0,
$$

with

$$
\alpha_{-}^{0}=\alpha_{00}^{0} \quad \text { and } \quad \alpha_{y-}^{0}=0 \quad \text { if } \lim _{y \rightarrow 0-} \frac{k}{k^{\prime}}=0 .
$$

A simple calculation shows that all of the above requirements in $G^{-}-(4.6)-(4.8)$ and (4.16)-(4.18) - are satisified if in $G^{-}$we have

$$
8 \lambda(-k)^{3 / 2} /\left(k^{\prime}\right)^{2}+1+2\left(k / k^{\prime}\right)^{\prime}>0 .
$$

Choosing $\alpha^{0}(y)=\alpha_{00}^{0}(<0), \alpha^{1}=\alpha^{2}=0$ in $G^{+}$, we see at once that condition (4.2) on $G \cap\{y=0\}$ and condition (3.15) on $\Gamma_{0}$ are satisifed. Thus from (4.1) we obtain

$$
\begin{aligned}
& A_{11}=-2 \alpha_{00}^{0} k(y) \geqslant 0, \quad A_{12}=0, \quad A_{22}=-2 \alpha^{0}>0, \\
& A_{00}=2 \lambda \alpha^{0}-\rho_{2}\left(\alpha^{2}\right)^{2} \geqslant d_{0}>0 .
\end{aligned}
$$

Hence we have

THEOREM 5.1. If

(1) $\operatorname{sgn} k(y)=\operatorname{sgn} y, \quad k(y) \in C^{0}(\bar{G}) \cap C^{2}(\bar{G} \cap\{y<0\}), \quad k^{\prime}(y)>0$ in $\bar{G} \cap$ $\{y<0\}$,

$$
\lim _{y \rightarrow 0-}\left(1+2\left(\frac{k}{k^{\prime}}\right)^{\prime}\right)>0, \quad \lim _{y \rightarrow 0-} \frac{k(y)}{k^{\prime}(y)}=0, \quad 8 \lambda \frac{(-k)^{3 / 2}}{\left(k^{\prime}\right)^{2}}+1+2\left(\frac{k}{k^{\prime}}\right)^{\prime}>0
$$

in $\bar{G}$

(2) $f(x, y) \in L^{2}(G), \lambda<0$,

then there exist $a$ weak and semistrong solution $u \in L^{2}(G)$ of the boundary value problem (1.4).

REMARK. If (1.4) has a strong solution, from our a priori estimates it follows that this solution is unique. For the special case when $k(y)=\operatorname{sgn} y|y|^{m}, m>0$, in (1.4), it is easy to show that (5.3) is stronger than (4.20). For $m>2$ if the functions $\alpha^{1}, \alpha^{2}$, $\alpha^{0}$ are chosen as in (4.21) and (4.22), the conclusion of Theorem 5.1 holds for (1.4) if $\lambda<0$ and $f \in L^{2}(G)$.

\section{REFERENCES}

I. S. Agmon, L. Nirenberg and M. H. Protter, A maximum principle for a class of hyperholic equations and applications to equations of mixed elliptic-hyperbolic type, Comm. Pure Appl. Math. 6 (1953), 455-470.

2. Ju. M. Berezanskii, Expansions in eigenfunctions of selfadjoint operators, Transl. Math. Monos., vol. 17, Amer. Math. Soc., Providence, R. I., 1968.

3. A. V. Bitsadze, Equations of the mixed type, Pergamon, New York, 1964.

4. S. Gellerstedt, Quelques problemes mixtes pour l'equation $y^{\prime m} z_{x x}+z_{1 W}=0$, Ark. Mat. Astr. Fysik 26A (1937).

5. S. G. Mikhlin, Linear equations of mathematical physics, Holt, Rinehart and Winston. New York, 1977.

6. C. S. Morawetz, A weak solution for a system of equations of elliptic-hyperbolic type, Comm. Pure Appl. Math. 11 (1958), 315-331. 
7. M. H. Protter, An existence theorem for the generalized Tricomi problem, Duke Math. J. 21 (1954), $1-7$.

8. M. Schneider, Funktionalanalytische Methoden zur Behandlung von linearen partiellen Differentialgleichungen vom gemischten Typ, Überblicke Mathematik, Bd. 7, Bibliographisches Inst., Mannheim, 1974, pp. 219-265.

9. . Uber schwache und halbstarke Lösungen des Tricomi-Problems, Math. Nachr. 60 (1974), $167-180$.

Department of Mathematics, University of Maryland, Baltimore, Maryland 21228

Institute for Physical Science and Technology, University of Maryland, College Park, MARYLAND 20742 